University of Nebraska - Lincoln

DigitalCommons@University of Nebraska - Lincoln

\title{
Metallic and Insulating Oxide Interfaces Controlled by Electronic Correlations
}

\author{
H. W. Jang \\ University of Wisconsin - Madison \\ D. A. Felker \\ University of Wisconsin - Madison \\ C. W. Bark \\ University of Wisconsin - Madison \\ Y. Wang \\ University of Nebraska-Lincoln \\ Manish K. Niranjan \\ University of Nebraska-Lincoln, manish@iith.ac.in \\ See next page for additional authors
}

Follow this and additional works at: https://digitalcommons.unl.edu/physicstsymbal

Part of the Condensed Matter Physics Commons

Jang, H. W.; Felker, D. A.; Bark, C. W.; Wang, Y.; Niranjan, Manish K.; Nelson, C. T.; Zhang, Y.; Su, D.; Folkman, C. M.; Baek, S. H.; Lee, S.; Janicka, K.; Zhu, Y.; Pan, X. Q.; Fong, D. D.; Tsymbal, Evgeny Y.; Rzchowski, M. S.; and Eom, Chang-Beom, "Metallic and Insulating Oxide Interfaces Controlled by Electronic Correlations" (2011). Evgeny Tsymbal Publications. 38.

https://digitalcommons.unl.edu/physicstsymbal/38

This Article is brought to you for free and open access by the Research Papers in Physics and Astronomy at DigitalCommons@University of Nebraska - Lincoln. It has been accepted for inclusion in Evgeny Tsymbal Publications by an authorized administrator of DigitalCommons@University of Nebraska - Lincoln. 


\section{Authors}

H. W. Jang, D. A. Felker, C. W. Bark, Y. Wang, Manish K. Niranjan, C. T. Nelson, Y. Zhang, D. Su, C. M.

Folkman, S. H. Baek, S. Lee, K. Janicka, Y. Zhu, X. Q. Pan, D. D. Fong, Evgeny Y. Tsymbal, M. S. Rzchowski, and Chang-Beom Eom 


\section{Metallic and Insulating Oxide Interfaces Controlled by Electronic Correlations}

H. W. Jang, ${ }^{1}$ D. A. Felker, ${ }^{2}$ C. W. Bark, ${ }^{1}$ Y. Wang, ${ }^{3}$ M. K. Niranjan, ${ }^{3}$ C. T. Nelson, ${ }^{4}$ Y. Zhang, ${ }^{4,5}$ D. Su, ${ }^{6}$ C. M. Folkman, ${ }^{1}$ S. H. Baek, ${ }^{1}$ S. Lee, ${ }^{1}$ K. Janicka, ${ }^{3}$ Y. Zhu, ${ }^{6}$ X. Q. Pan, ${ }^{4}$ D. D. Fong, ${ }^{7}$ E. Y. Tsymbal, ${ }^{3}$ M. S. Rzchowski, ${ }^{2}$ C. B. Eom ${ }^{1 *}$

The formation of two-dimensional electron gases (2DEGs) at complex oxide interfaces is directly influenced by the oxide electronic properties. We investigated how local electron correlations control the 2DEG by inserting a single atomic layer of a rare-earth oxide $(R O)$ [ $(R$ is lanthanum $(\mathrm{La})$, praseodymium $(\mathrm{Pr})$, neodymium $(\mathrm{Nd})$, samarium $(\mathrm{Sm})$, or yttrium $(\mathrm{Y})$ ] into an epitaxial strontium titanate oxide $\left(\mathrm{SrTiO}_{3}\right)$ matrix using pulsed-laser deposition with atomic layer control. We find that structures with $\mathrm{La}, \mathrm{Pr}$, and $\mathrm{Nd}$ ions result in conducting $2 \mathrm{DEGs}$ at the inserted layer, whereas the structures with Sm or $\mathrm{Y}$ ions are insulating. Our local spectroscopic and theoretical results indicate that the interfacial conductivity is dependent on electronic correlations that decay spatially into the $\mathrm{SrTiO}_{3}$ matrix. Such correlation effects can lead to new functionalities in designed heterostructures.

A dvanced deposition techniques enable the growth of epitaxial heterostructures with atomically controlled interfaces such as multilayers (1), superlattices (2-4), and ultrathin films $(5,6)$. In these artificial structures, the interfaces play a prominent role in determining the functionalities of the structures and their applications (7). A recent example is the discovery of two-dimensional electron gases (2DEGs) at the interface between complex insulating oxides $(8)$ such as $\mathrm{LaAlO}_{3} / \mathrm{SrTiO}_{3}(9,10), \mathrm{LaTiO}_{3} / \mathrm{SrTiO}_{3}$ (2), and $\mathrm{LaVO}_{3} / \mathrm{SrTiO}_{3}$ (11) heterostructures, in

${ }^{1}$ Department of Materials Science and Engineering, University of Wisconsin-Madison, Madison, WI 53706, USA. ²Department of Physics, University of Wisconsin-Madison, Madison, WI 53706, USA. ${ }^{3}$ Department of Physics and Astronomy, Nebraska Center for Materials and Nanoscience, University of NebraskaLincoln, Lincoln, NE 68588, USA. ${ }^{4}$ Department of Materials Science and Engineering, University of Michigan-Ann Arbor, Ann Arbor, MI 48109, USA. ${ }^{5}$ National Laboratory of Solid State Microstructures and Department of Materials Science and Engineering, Nanjing University, Nanjing 210093, P.R. China. ${ }^{6}$ Center for Functional Nanomaterials, Brookhaven National Laboratory, Upton, NY 11973, USA. ${ }^{7}$ Materials Science Division, Argonne National Laboratory, Argonne, IL 60439, USA. *To whom correspondence should be addressed. E-mail: eom@engr.wisc.edu. which the $2 \mathrm{DEG}$ is confined near the $\mathrm{LaO} / \mathrm{TiO}_{2}$ interface. Magnetic and superconducting ground states of the 2DEG have been identified (12-14),
A
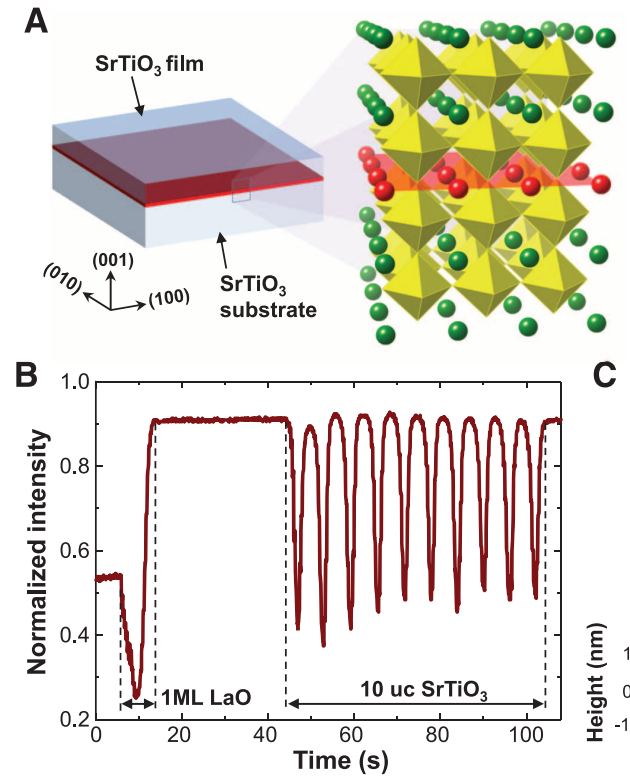

and applications to field-effect transistors and tunnel junctions have been demonstrated (15-17).

Theoretical work on $\mathrm{LaTiO}_{3} / \mathrm{SrTiO}_{3}$ superlattices (18) suggests that for a several-unit-cellthick $\mathrm{LaTiO}_{3}$ layer, the $\mathrm{LaTiO}_{3} / \mathrm{SrTiO}_{3}$ interface region is metallic; however, nonmetallic behavior dominates in the $\mathrm{LaTiO}_{3}$ region away from the interface, resulting from strong electron correlations similar to those found in bulk $\mathrm{LaTiO}_{3}$. In other bulk rare-earth titanates, the effect of electron correlations depends critically on the rareearth ion (19). We used the unique electronic charof their structure and composition, to deliberately manipulate the 2DEG electronic properties.

We studied the effect of strong electron correlations on an oxide 2DEG by inserting a single atomic layer of $R \mathrm{O}(R$ is $\mathrm{La}, \mathrm{Pr}, \mathrm{Nd}, \mathrm{Sm}$, or $\mathrm{Y}$ ) into an epitaxial $\mathrm{SrTiO}_{3}$ matrix using pulsed-laser deposition with atomic layer control. The $R \mathrm{O}$ layer donates electrons to the conduction band of $\mathrm{SrTiO}_{3}$. These electrons remain near the inserted $R \mathrm{O}$ layer due to Coulomb attraction. We find that the transport properties of these electrons range from metallic to insulating, depending critically acter of oxide interfaces, and atomic level control

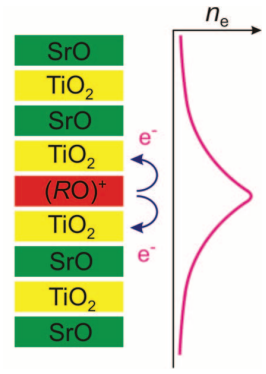

C

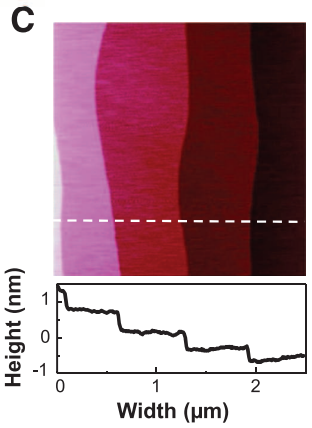

Fig. 1. (A) Schematic representation of a $\mathrm{SrTiO}_{3} /$ 1-ML $\mathrm{RO} / \mathrm{SrTiO}_{3}$ heterostructure. The atomic structure near the interface is enlarged. The +1 valent $R O$ layer donates electrons to neighboring $\mathrm{TiO}_{2}$ planes, leading to the larger electron density $n_{\mathrm{e}}$ near the interface. (B) Typical RHEED oscillations for the growth of 1-ML $\mathrm{LaO}$ and $10-\mathrm{uc} \mathrm{SrTiO}_{3}$ layers in sequence on a $\mathrm{TiO}_{2}^{-}$ terminated $\mathrm{SrTiO}_{3}$ substrate. (C) AFM image of a 10-uc $\mathrm{SrTiO}_{3} / 1-\mathrm{ML} \mathrm{LaO}_{\mathrm{SrTiO}}$ heterostructure showing an atomically smooth surface. 
on the rare-earth ion, and that this dependence arises from strong electronic correlations.

We grew epitaxial $\mathrm{SrTiO}_{3}$ heterostructures containing a symmetric $\mathrm{TiO}_{2} / R \mathrm{O} / \mathrm{TiO}_{2}$ interface (Fig. 1A), resulting in $\mathrm{RTiO}_{3}$-like structure at the interface. Using pulsed-laser deposition, the heterostructures were fabricated by depositing either a $\mathrm{RO}$ monolayer or a $\mathrm{RTiO}_{3}$ unit cell
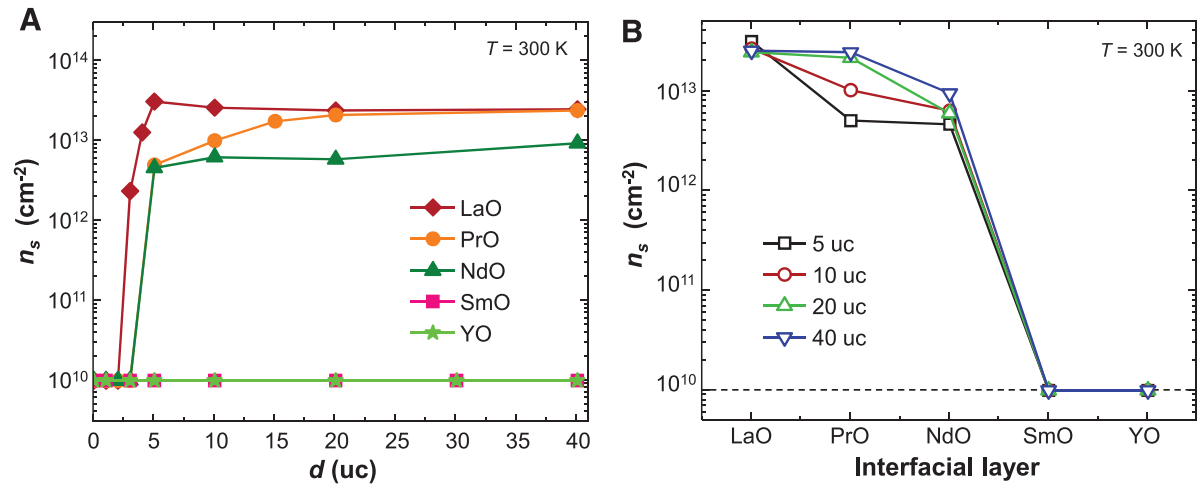

Fig. 2. Dependence of sheet carrier concentration $n_{5}$ on the $R$ ion in $\mathrm{SrTiO}_{3} / 1-\mathrm{ML} R \mathrm{O} / \mathrm{SrTiO}_{3}$ heterostructures and the $\mathrm{SrTiO}_{3}$ overlayer thickness $d$. Sheet carrier concentration is plotted as a function of (A) the thickness of the $\mathrm{SrTiO}_{3}$ overlayer and (B) the $R O$ doping layer for $\mathrm{SrTiO}_{3} / 1-\mathrm{ML} R O / \mathrm{SrTiO}_{3}$ heterostructures. SmO-based and YO-based heterostructures never become conducting, even with very thick $\mathrm{SrTiO}_{3}$ overlayers.
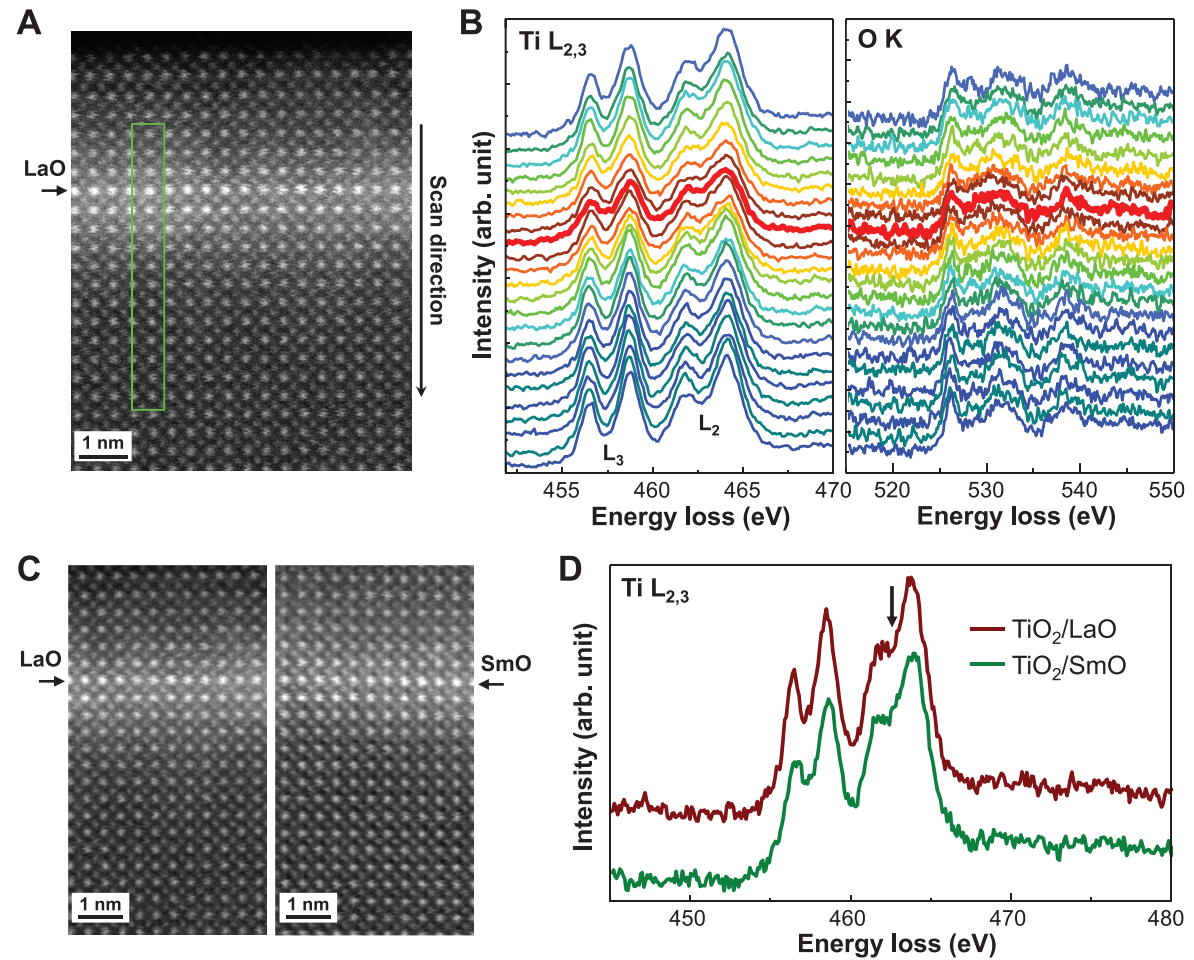

Fig. 3. STEM and EELS analysis. (A) High-angle annular dark field (HAADF) image of a 10-uc $\mathrm{SrTiO}_{3} / 1-\mathrm{ML}$ $\mathrm{LaO}$ film grown on $\mathrm{SrTiO}_{3}$. The rectangular box represents the region of EELS line scans. (B) EELS spectra of $T-L_{2,3}$ and $0-K$ edges obtained from $2 \mathrm{D}$ line scans across the interface shown in (A). The spacing along the line scan between consecutive EELS spectra is $2.8 \AA$. The spectra at the LaO layer are highlighted by thicker lines. For the spectra for Ti $L_{2}$ and $L_{3}$ edges, peak broadening and less pronounced peak splitting at the interface are clearly observed. (C) HAADF images of 10-uc $\mathrm{SrTiO}_{3} / 1-\mathrm{ML} \mathrm{LaO}_{\mathrm{SrTiO}}$ and $10-\mathrm{uc} \mathrm{SrTiO}_{3} / 1-\mathrm{ML}$ $\mathrm{SmO} / \mathrm{SrTiO}_{3}$ heterostructures. Both samples show no obvious defects or dislocations, indicating coherent interfaces. (D) Selected area Ti- $\mathrm{L}_{2,3}$ EELS spectra obtained at the interfaces for 10 -uc $\mathrm{SrTiO}_{3} / 1-\mathrm{ML} \mathrm{LaO} / \mathrm{SrTiO}_{3}$ and 10 -uc $\mathrm{SrTiO}_{3} / 1-\mathrm{ML} \mathrm{SmO} / \mathrm{SrTiO}_{3}$ heterostructures. The arrow is a guide for comparison. tron diffraction (RHEED) intensity oscillations. Typical RHEED oscillations for the growth of a 10 -uc $\mathrm{SrTiO}_{3} / 1-\mathrm{ML} \mathrm{LaO}$ heterostructure on a $\mathrm{SrTiO}_{3}$ substrate are shown in Fig. 1B. The atomic force microscopy (AFM) image of the surface of a complete heterostructure (Fig. 1C) shows the steps and terraces of the original substrate surface, indicating high-quality growth. Microstructure and electrical properties of both $\mathrm{SrTiO}_{3} / 1-\mathrm{ML}$ $R \mathrm{O} / \mathrm{SrTiO}_{3}$ and $\mathrm{SrTiO}_{3} / 1$-uc $R \mathrm{TiO}_{3} / \mathrm{SrTiO}_{3}$ heterostructures were almost identical (20). Here, we focus on the $\mathrm{SrTiO}_{3} / R \mathrm{O} / \mathrm{SrTiO}_{3}$ heterostructures.

We first characterized the dependence of electrical properties on growth conditions, using the LaO-based heterostructure, and established the growth conditions of oxygen pressure $\left(P_{\mathrm{O}_{2}}=\right.$ $\left.10^{-3} \mathrm{mbar}\right)$ and temperature $\left(T_{\text {growth }}=550^{\circ} \mathrm{C}\right)$ as optimal (20). These growth conditions were used to fabricate $\mathrm{SrTiO}_{3}$ heterostructures with single inserted atomic layers of $\mathrm{LaO}, \mathrm{PrO}, \mathrm{NdO}, \mathrm{SmO}$, and YO. Fig. 2A shows the mobile sheet carrier concentration $n_{s}$ for the five different $R \mathrm{O}$ layers as a function of the $\mathrm{SrTiO}_{3}$ overlayer thickness. It is seen that LaO-, PrO-, and NdO-based heterostructures become conducting above the critical thickness of $\mathrm{SrTiO}_{3}$ of three or four unit cells. However, SmO- and YO-based heterostructures are insulating, even with a $\mathrm{SrTiO}_{3}$ overlayer thickness of 100 uc. This is summarized in Fig. $2 \mathrm{~B}$, which shows the mobile sheet carrier concentration at fixed overlayer thickness as the rare-earth ion progresses from La to Y. The nominal room-temperature concentration of mobile carriers in crossover NdO-based heterostructures decreases dramatically at lower temperatures (fig. $\mathrm{S} 3 \mathrm{D}$ ), in contrast to the relatively temperatureindependent behavior of the conducting $\mathrm{LaO}$ based and PrO-based heterostructures. This trend is analogous to that in bulk $R \mathrm{TiO}_{3}$, where the effects of electron correlations increase as $R$ is varied from La to $\mathrm{Y}(21)$. The mobilities of all conducting heterostructures are roughly independent of the rare-earth ion, showing a crossover from temperature-dependent phonon scattering at high temperature to a temperature-independent value at low temperatures.

Our transport measurements are sensitive to mobile carriers near the interface. We also investigated charge transfer from the $R O$ layer to nearby $\mathrm{Ti}$ states with electron energy-loss spectroscopy (EELS), sensitive to both mobile and nonmobile carriers $(2,10)$. For a conducting $\mathrm{LaO}-$ based heterostructure, the spatial dependence of EELS spectra of Ti- $\mathrm{L}_{2,3}$ and O-K edges is shown in Fig. 3B. The EELS spectra are spatially separated by $0.28 \mathrm{~nm}$, in a line scan across the interface of a 10 -uc $\mathrm{SrTiO}_{3} / 1-\mathrm{ML} \mathrm{LaO} / \mathrm{SrTiO}_{3}$ heterostructure (Fig. 3A). Four clear peaks in the Ti $\mathrm{L}_{2,3}$ edge become broader at the interface, with peak separations less pronounced. We attribute this broadening to the presence of a $\mathrm{Ti}^{3+}$ component. Compared with previous reports $(2,10)$, the relatively small modulation of the EELS signal at the interface may be related to the low $n_{s}$ determined from the Hall effect. Our depth profil- 
ing of the $\mathrm{Ti}^{3+}$ to $\mathrm{Ti}^{4+}$ ratios indicates that the carriers are confined to within $\sim 1 \mathrm{~nm}$ of the interface (fig. S6), in good agreement with recent

Fig. 3, C and D, show scanning transmission electron microscope (STEM) images and selected area $\mathrm{Ti}-\mathrm{L}_{2,3}$ EELS spectra, at $\mathrm{TiO}_{2}$ planes adjacent to the interface, for $\mathrm{LaO}$ (conducting) and $\mathrm{SmO}$ (insulating) heterostructures. For both heterostructures, the STEM images and the Ti- $\mathrm{L}_{2,3}$ spectra at the interface look very similar. In particular, the very similar peak splittings at $\sim 462 \mathrm{eV}$ in the $\mathrm{Ti} \mathrm{L}_{2}$ edges suggest that the electron transfer from the $R O$ layer to the neighboring $\mathrm{TiO}_{2}$ planes is the same for both $\mathrm{LaO}$ - and SmO-based heterostructures. Our transport measurements indicate that these electrons produce a conducting 2DEG in $\mathrm{LaO}$ heterostructures but are not mobile in $\mathrm{SmO}$ heterostructures.

$\mathrm{TiO}_{6}$ octahedra rotations in bulk $R \mathrm{TiO}_{3}$ determine the width of the Ti- $3 d$ band of $t_{2 \mathrm{~g}}$ symmetry, and hence the electronic properties, through a change in the Mott-Hubbard gap (23). $\mathrm{SrTiO}_{3}$, however, has no $\mathrm{TiO}_{6}$ octahedral rotations at room temperature. We investigated octahedral rotations in our $\mathrm{SrTiO}_{3} / R \mathrm{O} / \mathrm{SrTiO}_{3}$ heterostructures, with synchrotron x-ray experiments at the Advanced Photon Source. We observed strong superlattice reflections (figs. S7 and S8) resulting from unitcell doubling $\mathrm{TiO}_{6}$ octahedra rotations, in good agreement with the density functional calculations discussed below. The octahedral rotations are well ordered in the interfacial plane, with typical rocking widths giving an in-plane domain size $>60 \mathrm{~nm}$. The breadths of the half-order peaks in the out-of-plane direction are consistent with octahedral rotations at the $R \mathrm{TiO}_{3}$ layer rapidly decaying into the $\mathrm{SrTiO}_{3}$ matrix. These decaying octahedra rotations lead to a spatial gradient in the electronic structure, influencing the conduction.

In addition, epitaxial strain in the interfacial $R \mathrm{TiO}_{3}$ layer also affects the interface conductivtheoretical calculations (22).

ity. $\mathrm{LaTiO}_{3}, \mathrm{PrTiO}_{3}$, and $\mathrm{NdTiO}_{3}$ layers at the interface are strained under biaxial compression, but $\mathrm{SmTiO}_{3}$ and $\mathrm{YTiO}_{3}$ layers are under biaxial tension (table S1) (21). Compressive strain has been shown to induce conducting behavior in $\mathrm{LaTiO}_{3}$ thin films (24), attributed to an increased Ti $t_{2 \mathrm{~g}}$ bandwidth and a weakened crystal field. This has been predicted theoretically to reduce the effect of electron correlations and to support metallic behavior (25). The tensile strain in the $\mathrm{SmTiO}_{3}$ and $\mathrm{YTiO}_{3}$ layers embedded in $\mathrm{SrTiO}_{3}$ appears to enforce the effect of strong correlations and favor insulating behavior.

To understand the combined effects of charge transfer, spatially varying octahedral rotations, biaxial strain, and rare-earth electronic structure, we have performed density functional theory (DFT) calculations, including a Hubbard $U$ term accounting for the on-site Coulomb interaction (20). The values of $U$ that provide a realistic description of the electronic and atomic structure of bulk $\mathrm{YTiO}_{3}$ and $\mathrm{LaTiO}_{3}$ compounds (26) were used. The atomic positions were fully relaxed, under the constraint that the in-plane lattice constant be equal to the calculated lattice constant of bulk $\mathrm{SrTiO}_{3}$. The density of electronic states, and the corresponding atomic coordinates, calculated for periodic superlattices, are shown in Fig. 4A (3.5-uc $\mathrm{SrTiO}_{3} / 1-\mathrm{ML} \mathrm{LaO}$ ) and in Fig. $4 \mathrm{~B}$ (3.5-uc $\mathrm{SrTiO}_{3} / 1-\mathrm{ML}$ YO). For the LaObased heterostructure, the Fermi energy lies in the region of nonzero density of states, consistent with the previous calculations $(27,28)$, whereas for the YO heterostructure the Fermi energy lies between the split-off lower Hubbard band and the higher energy density of states. This indicates that the LaO-based interface is metallic, whereas the YO-based interface is insulating, supporting our experimental observations. Our calculations predict that the ground state of the $\mathrm{SrTiO}_{3} / \mathrm{LaO}$ heterostructure is not chargeordered, whereas the $\mathrm{SrTiO}_{3} / \mathrm{YO}$ heterostructure

Fig. 4. Energy-dependent density of states and structural relaxation of 3.5-uc $\mathrm{SrTiO}_{3} / 1-\mathrm{ML} \mathrm{LaO}$ (A and C) periodic superlattice and 3.5-uc $\mathrm{SrTiO}_{3} / 1-\mathrm{ML}$ YO periodic superlattice (B and D) obtained from DFT calculations. Positive density of states is for spin up and negative is for spin down. The dashed line indicates the position of the Fermi level. The results indicate conducting behavior for the 3.5-uc $\mathrm{SrTiO}_{3} / 1-\mathrm{ML}$ $\mathrm{LaO}$ periodic superlattice and insulating behavior for the 3.5-uc $\mathrm{SrTiO}_{3} / 1-\mathrm{ML}$ YO periodic superlattice.

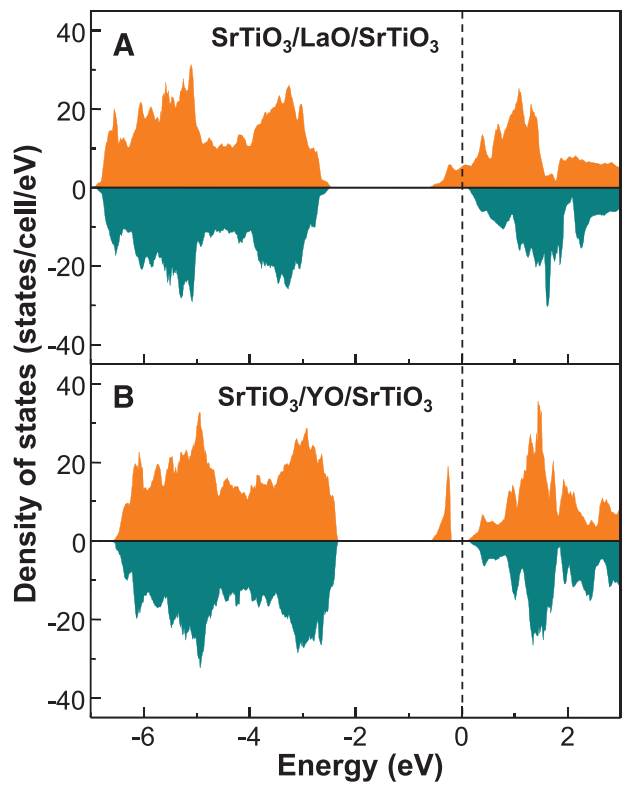

is unstable with respect to charge disproportionation and has a charge-ordered ground state similar to that found in (29). Octahedra rotations are clearly visible in the relaxed structures shown in Fig. 4, C and D, consistent with our synchrotron measurements.

The electron donated by the $\mathrm{RO}$ embedded in the $\mathrm{SrTiO}_{3}$ matrix is localized to the nearby $\mathrm{TiO}_{2}$ layers. Filling of the Ti-3d band in these layers close to $n=0.5$, and enhanced electroncorrelation effects due to $2 \mathrm{D}$ confinement, will strongly influence the interfacial conductivity. It is well known that the effect of Ti-3d band filling on electronic, magnetic, and transport properties of bulk $R \mathrm{TiO}_{3}$ Mott-Hubbard insulators depends critically on the rare-earth ion (23). It appears that for the relatively weakly correlated $\mathrm{LaO}$-based heterostructure, several percent of hole doping is sufficient to cause a metalinsulator transition. In contrast, for the YO-based heterostructures with larger $U$, lower bandwidth $W$, and larger strain and structural distortions, the insulating phase persists. The number of electrons transferred in each case is the same, but stronger correlation effects in the YO heterostructure seem to be responsible for the insulating behavior. Our experimental and theoretical investigations suggest that these correlations arise from an interplay of strain, spatially varying rotational distortions, and rare-earth ion effects on the band structure. Indications of electron correlations have also been recently reported in $\mathrm{LaIO}_{3} / \mathrm{SrTiO}_{3}$ heterostructures (30).

Strong correlations in 2DEGs at oxide interfaces have been shown to result from electronic properties of different $R \mathrm{O}$ inserted layers, as well as the structural and electronic modification of nearby layers. Quantitatively exploring the underlying physics of the experimental data presented here is complex and challenging, because strong correlations combined with atomic-scale structural and chemical variations severely limit the effectiveness of theoretical calculations. The details cannot be fully captured within the DFT $+\mathrm{U}$ calculations used here, and more advanced approachesbased on dynamical mean-field theory (31), for example - are likely necessary to capture the spatial variations. The work presented here is important in elucidating correlation effects in systems with atomic-scale perturbations (32) and external perturbation-induced changes in oxide 2DEG systems $(8,15-17)$. The ability to design and grow heterostructures with atomic-scale variations, and the demonstrated strong dependence of correlated 2DEGs on these variations, open new directions for oxide 2DEG heterostructures.

References and Notes

1. H. Yamada et al., Science 305, 646 (2004).

2. A. Ohtomo, D. A. Muller, ]. L. Grazul, H. Y. Hwang, Nature 419, 378 (2002).

3. M. P. Warusawithana, E. V. Colla, ]. N. Eckstein M. B. Weissman, Phys. Rev. Lett. 90, 036802 (2003).

4. E. Bousquet et al., Nature 452, 732 (2008).

5. M. P. Warusawithana et al., Science 324, 367 (2009). 
6. V. Garcia et al. Nature 460, 81 (2009).

7. H. Kroemer, Surf. Sci. 132, 543 (1983).

8. ]. Mannhart, D. G. Schlom, Science 327, 1607 (2010).

9. A. Ohtomo, H. Y. Hwang, Nature 427, 423 (2004).

10. N. Nakagawa, H. Y. Hwang, D. A. Muller, Nat. Mater. 5, 204 (2006).

11. Y. Hotta, T. Susaki, H. Y. Hwang, Phys. Rev. Lett. 99, 236805 (2007)

12. A. Brinkman et al., Nat. Mater. 6, 493 (2007).

13. N. Reyren et al., Science 317, 1196 (2007).

14. Y. Kozuka et al., Nature 462, 487 (2009).

15. S. Thiel, G. Hammerl, A. Schmehl, C. W. Schneider, ]. Mannhart, Science 313, 1942 (2006)

16. C. Cen et al., Nat. Mater. 7, 298 (2008).

17. C. Cen, S. Thiel, ]. Mannhart, ]. Levy, Science 323, 1026 (2009).

18. S. Okamoto, A. J. Millis, Nature 428, 630 (2004).

19. Y. Okimoto, T. Katsufuji, Y. Okada, T. Arima, Y. Tokura, Phys. Rev. B 51, 9581 (1995).

20. Materials and methods are available as supporting material on Science Online.

21. H. D. Zhou, J. B. Goodenough, J. Phys. Condens. Matter 17, 7395 (2005)

22. K. Janicka, J. P. Velev, E. Y. Tsymbal, Phys. Rev. Lett. 102 106803 (2009).
23. M. Imada, A. Fujimori, Y. Tokura, Rev. Mod. Phys. 70, 1039 (1998).

24. F. J. Wong et al., Phys. Rev. B 81, 161101 (2010).

25. H. Ishida, A. Liebsch, Phys. Rev. B 77, 115350 (2008).

26. S. Okatov, A. Poteryaev, A. Lichtenstein, Europhys. Lett. 70, 499 (2005).

27. Z. S. Popovic, S. Satpathy, Phys. Rev. Lett. 94, 176805 (2005).

28. S. Okamoto, A. J. Millis, N. A. Spaldin, Phys. Rev. Lett. 97, 056802 (2006).

29. R. Pentcheva, W. E. Pickett, Phys. Rev. Lett. 99, 016802 (2007).

30. M. Breitschaft et al., Phys. Rev. B 81, 153414 (2010).

31. G. Kotliar et al., Rev. Mod. Phys. 78, 865 (2006).

32. Q. Si, M. J. Rozenberg, G. Kotliar, A. E. Ruckenstein, Phys. Rev. Lett. 72, 2761 (1994).

33. We thank D. G. Schlom and D. A. Muller for fruitful discussions. This work was supported by the National Science Foundation under grant DMR-0906443 and a David and Lucile Packard Fellowship (C.B.E.). The research at University of Nebraska was supported by the Materials Research Science and Engineering Center (NSF grant DMR-0820521), the Nanoelectronics Research Initiative of the Semiconductor Research Corporation, the National Science Foundation (grant
EPS-1010674), and the Nebraska Research Initiative. Work at the University of Michigan was supported by the U.S. Department of Energy (DOE) under grant DE-FG02-07ER46416. We thank the National Center for Electron Microscopy at Lawrence Berkeley National Laboratory for their support under DOE grant DE-AC02-05CH11231 for user facilities. Work at Argonne and use of the Advanced Photon Source were supported by the DOE Office of Science, Office of Basic Energy Sciences, under contract DE-AC0206CH11357. Work at Brookhaven National Laboratory was sponsored by DOE/BES/MSE and the Center for Functional Nanomaterials under contract DE-ACO298CH10886. J. Karapetrova's assistance at beamline 33-BM of the Advanced Photon Source is gratefully acknowledged.

\section{Supporting Online Material}

www.sciencemag.org/cgi/content/full/331/6019/886/DC1

Materials and Methods

Figs. $\mathrm{S} 1$ to $\mathrm{S} 8$

Table S1

References

7 October 2010; accepted 19 January 2011

10.1126/science.1198781 


\section{Science \\ \IAAAS}

\section{Supporting Online Material for}

\section{Metallic and Insulating Oxide Interfaces Controlled by Electronic Correlations}

H. W. Jang, D. A. Felker, C. W. Bark, Y. Wang, M. K. Niranjan, C. T. Nelson, Y. Zhang, D. Su, C. M. Folkman, S. H. Baek, S. Lee, K. Janicka, Y. Zhu, X. Q. Pan, D. D. Fong, E. Y. Tsymbal, M. S. Rzchowski, C. B. Eom*

*To whom correspondence should be addressed. E-mail: eom@engr.wisc.edu

Published 18 February 2011, Science 331, 886 (2011)

DOI: 10.1126/science.1198781

\section{This PDF file includes:}

Materials and Methods

Figs. S1 to S8

Table S1

References 


\section{Materials and methods}

\section{Growth}

All heterostructures were grown on $\mathrm{TiO}_{2}$-terminated $\mathrm{SrTiO}_{3}$ substrates by pulsed laser deposition (PLD) in an oxygen atmosphere with in-situ reflection high-energy electron diffraction (RHEED). Two types of heterostructures were grown. In the first, we deposited a $R O$ monolayer from a $R_{2} \mathrm{O}_{3}$ target, followed by deposition of a $\mathrm{SrTiO}_{3}$ overlayer. In the second we directly deposited a $R \mathrm{TiO}_{3}$ unit cell from a $R \mathrm{TiO}_{3+\mathrm{x}}$ target, followed by deposition of a $\mathrm{SrTiO}_{3}$ overlayer. Both types of heterostructures were grown with five different rare-earth ions $(R=\mathrm{La}$, Pr, Nd, Sm, Y), and with various $\mathrm{SrTiO}_{3}$ overlayer thicknesses varying from 0 to 40 unit cells. Heterostructures of both types with $R=$ La were grown at various oxygen pressures from $10^{-6}$ mbar to 0.1 mbar at a fixed growth temperature of $500{ }^{\circ} \mathrm{C}$, and growth temperatures from $500{ }^{\circ} \mathrm{C}$ to 800 at a fixed growth pressure of $10^{-3}$ mbar to determine optimal growth parameters of $T=$ 500-550 ${ }^{\circ} \mathrm{C}$ and $P_{\mathrm{O}_{2}}=10^{-3} \mathrm{mbar}$. The laser energy density of $2 \mathrm{~J} / \mathrm{cm}^{2}$ and the repetition rate of $2-3 \mathrm{~Hz}$ were used. The distance from the target surface to the sample was $55 \mathrm{~mm}$ and the growth rate of the film was 25-35 pulses per unit cell.

\section{Electrical measurements}

Electrical characteristics of most samples were measured with the van der Pauw technique. Ohmic contacts onto four corners of a $5 \mathrm{~mm} \times 5 \mathrm{~mm}$ sample were formed using Al wedge bonding directly connected to the sample surface. Comparison samples with wire bonding to ionbeam etched, metalized contacts in a van der Pauw geometry did not show substantial difference. Samples patterned in a Hall configuration also showed similar electrical characteristics. The data presented in this report were extracted from van der Pauw measurements with leads wire bonded directly to the sample surface. Nominal sheet carrier concentration $n_{s}$ and carrier mobility $\mu$ were determined as $n_{s}=H / e R_{H}$ and $\mu=R_{H} / H R_{\square}$, where $R_{H}$ is the Hall resistance, $R_{\square}$ the sheet resistance, $H$ the magnetic field applied perpendicular to the substrate, and $e$ the magnitude

of the electron charge. This assumes a single band of carriers, and a spherical Fermi surface. 
Figure S2A shows the dependence of nominal sheet carrier density $\left(n_{s}\right)$ and mobility $(\mu)$ on

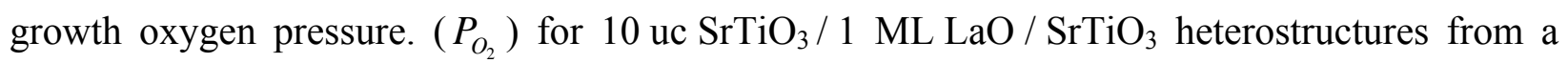
basic interpretation of the Hall measurements. The dependence of $n_{s}$ on $P_{\mathrm{O}_{2}}$ is significant, whereas $\mu$ is insensitive to $P_{O_{2}}$. The decrease of $n_{s}$ with increasing $P_{O_{2}}$ is consistent with the previous reports for $\mathrm{LaAlO}_{3} / \mathrm{SrTiO}_{3}$ system $(S 1, S 2, S 3, S 4, S 5)$. For $P_{O_{2}} \geq 10^{-3} \mathrm{mbar}$, carrier concentrations as low as $10^{13} \mathrm{~cm}^{-2}$, which is believed to be an intrinsic feature of the conducting $\mathrm{LaO} / \mathrm{TiO}_{2}$ interface $(S 1, S 5, S 6, S 7, S 8)$, can be obtained without additional post annealing. In contrast to $P_{O_{2}}$, we found that there is no notable dependence of $n_{s}$ and $\mu$ on growth temperature

(Fig. S1). A 10 uc $\mathrm{SrTiO}_{3} / 1 \mathrm{ML} \mathrm{LaO} / \mathrm{SrTiO}_{3}$ heterostructure grown at $P_{O_{2}}=10^{-3}$ mbar shows almost constant $n_{s}$ from $3 \mathrm{~K}$ to $300 \mathrm{~K}$ in Fig. S2B. The measured $\mu$ of $1250 \mathrm{~cm}^{2} \mathrm{~V} / \mathrm{s}$ at $3 \mathrm{~K}$ is similar to that previously reported for oxidized $\mathrm{LaAlO}_{3} / \mathrm{SrTiO}_{3}$ heterostructures (S5).

\section{First principles calculations}

First-principles electronic structure calculations have been performed within density functional theory (S9) applied to $3.5 \mathrm{uc} \mathrm{SrTiO}_{3} / 1 \mathrm{ML} \mathrm{MO}(\mathrm{M}=\mathrm{La}, \mathrm{Y})$ superlattices. The calculations employed the projector augmented wave (PAW) method (S10) as implemented in the Vienna Ab Initio Simulation Package (VASP) code (S11) The exchange-correlation effects were treated within generalized gradient approximation (GGA). The calculations were carried out using a plane-wave basis set limited by a cutoff energy of $520 \mathrm{eV}$ and the $6 \times 6 \times 4$ mesh of $\mathrm{k}$ points in the irreducible Brillouin zone with energy converged to $10^{-5} \mathrm{eV} / \mathrm{cell}$. Atomic relaxations were performed until the Hellmann-Feynman forces on atoms have become less than $10 \mathrm{meV} / \AA$. The lateral unit cells of the structures were constrained to have $c(2 \times 2)$ symmetry and the in-plane lattice constant to be the calculated bulk lattice constant of $\mathrm{SrTiO}_{3}$.

Electron-electron correlations of the partially filled $\mathrm{Ti} 3 \mathrm{~d}$-states were treated within the GGA+U approach (S12) that involves a Hubbard U term responsible for the on-site Coulomb interaction. The values of $U$ were chosen to provide a reasonable agreement between the calculated and measured optical band gaps for bulk $\mathrm{LaTiO}_{3}$ and $\mathrm{YTiO}_{3}$ compounds. Thus, for the $\mathrm{LaO}$-based structure the value of $\mathrm{U}$ was chosen to be $3 \mathrm{eV}$, while for the YO-based structure the 
value of $\mathrm{U}$ was fixed at $4 \mathrm{eV}$. The calculated electronic structures of bulk $\mathrm{LaTiO}_{3}$ and $\mathrm{YTiO}_{3}$ are in agreement with previous calculations (S13) and describe correctly the atomic structures and the ground states of these compounds. The smaller $\mathrm{U}$ in the case of $\mathrm{LaTiO}_{3}$ as compared to $\mathrm{YTiO}_{3}$ is justified (S14) since the bandwidth of the Ti $3 \mathrm{~d}$ states in $\mathrm{LaTiO}_{3}$ is smaller than that in $\mathrm{YTiO}_{3}$ due to larger $\mathrm{GdFeO}_{3}$-type distortions for the latter. This is found to be also the case for the $3.5 \mathrm{uc} \mathrm{SrTiO}_{3} / 1 \mathrm{ML}$ MO superlattices where the octahedral distortions are much more pronounced for $\mathrm{M}=\mathrm{Y}$ than for $\mathrm{M}=\mathrm{La}$ (see Figs. 4C, 4D). In particular, the tilting angles of the Ti-O-Ti bonds along the [001] axis for the oxygen octahedra ending at the $\mathrm{M}$ atom are $147^{\circ}$ for $\mathrm{M}=\mathrm{Y}$ as compared to $162^{\circ}$ for $\mathrm{M}=\mathrm{La}$. Since the $\mathrm{La} 4 \mathrm{f}$ bands lie at higher energy than that predicted by GGA, the value of $\mathrm{U}$ for the $\mathrm{La} 4 \mathrm{f}$ states was taken to be $8.0 \mathrm{eV}$ to avoid their spurious mixing with the conduction bands. The value of the exchange parameter was fixed at $\mathrm{J}=1 \mathrm{eV}$ since its value is almost independent of the choice of materials.

Including octahedral distortions in the structural model is essential (S15) and leads to a significant reduction in energy for the considered $c(2 \times 2)$ structure as compared to the $p(1 \times 1)$ structure (S16). For the values of $\mathrm{U}$ chosen, we have that this energy difference is about $0.42 \mathrm{eV}$ per equivalent $(1 \times 1)$ lateral cell for the $\mathrm{LaO}$-monolayer system and $1.38 \mathrm{eV}$ for the YOmonolayer system. Our calculations also showed that while the ground state of the $\mathrm{SrTiO}_{3} / \mathrm{LaO}$ heterostructure is non-charge ordered, the $\mathrm{SrTiO}_{3} / \mathrm{YO}$ heterostructure is unstable with respect to charge disproportionation and has a charged-ordered ground state (S17). Within the $\mathrm{c}(2 \times 2)$ structure constraint the latter is a ferromagnetic insulator characterized by two non-equivalent $\mathrm{Ti}$ atoms in the plane of the structure with magnetic moments of $0.90 \mu_{\mathrm{B}}$ and $0.05 \mu_{\mathrm{B}}$ at the interface layer and nearly zero magnetic moments away from the interface. The charge and orbital ordering are similar to those found in ref. (S17) and characterized by charge disproportionation $\mathrm{Ti}^{3.9+} / \mathrm{Ti}^{3.05+}$ with preferential $\mathrm{Ti}^{3.05+} d_{x y}$-orbital occupation at the interface. The gain in energy for the charge ordered configuration as compared to the non-charge ordered configuration for the $\mathrm{SrTiO}_{3} / \mathrm{YO}$ system is about $0.14 \mathrm{eV}$ per equivalent $(1 \times 1)$ lateral cell. For $\mathrm{SrTiO}_{3} / \mathrm{MO}$ heterostructures with smaller $\mathrm{SrTiO}_{3}$ thickness of $1.5 \mathrm{uc}$ we also checked a possibility of the $p(2 \times 2)$ charge-ordered anti-ferromagnetic state. We found that the total energy of this state is higher by about $0.11 \mathrm{eV}$ for the $\mathrm{SrTiO}_{3} / \mathrm{LaO}$ structure, while for the $\mathrm{SrTiO}_{3} / \mathrm{YO}$ structure it is lower by $12 \mathrm{meV}$, however, the latter value is close to the accuracy of our computations. 


\section{$\mathrm{SrTiO}_{3}$ overlayer critical thickness}

Additional experimental insight comes from our measured dependence on the $\mathrm{SrTiO}_{3}$ overlayer thickness. We have found that although a $\mathrm{LaO}$ layer embedded in $\mathrm{SrTiO}_{3}$ is conducting, a $1 \mathrm{ML} \mathrm{LaO}$ film $(d=0 \mathrm{uc})$ on $\mathrm{SrTiO}_{3}$ is insulating. The film is still insulating with a 2 uc $\mathrm{SrTiO}_{3}$ overlayer, but becomes conducting with a $3 \mathrm{uc} \mathrm{SrTiO}_{3}$ overlayer, corresponding to the critical thickness $\left(d_{c}\right)$ of 3 uc. We obtained similar results for different rare earth ions (see Fig. S3A and $\mathrm{S} 3 \mathrm{~B})$. We measured the $n_{s}$ of $\mathrm{SrTiO}_{3} / 1 \mathrm{ML} \mathrm{LaO}$ films on $\mathrm{SrTiO}_{3}$ with increasing thickness of $\mathrm{SrTiO}_{3}$ overlayer $(d)$. For $d \geq 5 \mathrm{uc}$, the film shows a saturation in $n_{s}$, which means that the conductance is confined to a narrow thickness range of the sample, that is, a 2DEG. This could be explained by a change in the Ti-3d band filling. In the absence of the $\mathrm{SrTiO}_{3}$ overlayer an extra electron provided by the $\mathrm{LaO}$ is localized close to the surface making the band filling of the interfacial $\mathrm{TiO}_{2}$ monolayer close to $n=1$ as in bulk insulating $\mathrm{LaTiO}_{3}$.

Depositing $\mathrm{SrTiO}_{3}$ on top of $\mathrm{LaO}$ leads to a redistribution of electron density between the top and bottom $\mathrm{TiO}_{2}$ layers, reducing the $\mathrm{Ti} 3 d$ band occupation and resulting in the transition to a metallic state, as discussed previously in a different context. Our calculations predict, however, that one unit cell of the $\mathrm{SrTiO}_{3}$ overlayer is sufficient to cause a metal-insulator transition in the system, somewhat inconsistent with our 3 uc experimental $\mathrm{SrTiO}_{3}$ critical thickness. This may be due to limitations of the DFT calculations, or to the presence of adsorbates on the surface neglected in our structural model. Alternatively, surface adsorbates providing low-energy states could pull electrons from the $R \mathrm{O}$ monolayer, producing insulating behavior for small (or zero) $\mathrm{SrTiO}_{3}$ overlayer thicknesses. The electric field from the resulting charge separation would raise the surface state energies with increasing $\mathrm{SrTiO}_{3}$ thickness, leading to repopulation of the $R \mathrm{O}$ interface above a critical $\mathrm{SrTiO}_{3}$ thickness.

\section{STEM and EELS analysis}

Transmission electron microscopy studies were performed using an aberration corrected scanning transmission electron microscope (STEM), Hitachi HD2700C, equipped with a coldfield-emission electron source and a high-resolution electron energy-loss spectrometer. Crosssectional cuts of the samples grown under the optimum condition described above were prepared 
by mechanical polishing followed by low-energy and low-angle ion milling. For high angle annular dark field (HAADF) imaging, a probe size of $0.8-1 \AA$, a convergence angle of $28 \mathrm{mrad}$, and a HAADF collection angle of 114-608 mrad were used. For electron energy-loss spectroscopy (EELS) in STEM, a probe size of $1.3 \AA$, a convergence angle of $28 \mathrm{mrad}$, a HAADF collection angle of 45-242 mrad, and an EELS collection angle of $20 \mathrm{mrad}$ were used. For a beam current of 50-100 pA the acquisition time for EELS is about 1-2 sec and the energy resolution is about $0.4 \mathrm{eV}$.

The challenge for atomically resolved EELS study of the heterostructures is to minimize radiation damage on the sample. Our study shows that the electron illumination degrades the valence state of $\mathrm{Ti}$ from $\mathrm{Ti}^{4+}$ to $\mathrm{Ti}^{3+}$. Therefore, in this study, to obtain good signal-noise ratio spectra and avoid the artifact from the beam damage, we used the selected area EELS and 2D scanning methods with proper probe currents (around $50 \mathrm{pA}$ ) and scanning step ( $\geq$ the probe size). The quality of the data were monitored from the HAADF image (Fig. S4) as well as from the EELS spectra, which were then compared with those at the $\mathrm{SrTiO}_{3}$ substrate far away from the interface to ensure the spectra acquired are damage-free. The line scanning spacing between each spectrum is $1.2-1.6 \AA$. The 2D EELS scanning spectra presented in the manuscript were selected every other spectrum from the raw data. The selected area EELS spectra of $\mathrm{Ti}_{2,3}$ edge were normalized by the peak height of the $\mathrm{Ti}_{2}$ edges.

Thick films of the reference samples of $\mathrm{LaTiO}_{3}\left(\mathrm{Ti}^{3+}\right)$ and $\mathrm{SrTiO}_{3}\left(\mathrm{Ti}^{4+}\right)$ were prepared and measured. The EELS data was analyzed using the method described in Ref. 2 of the main text. The experimental spectrum at the $\mathrm{LaO}$ layer was fit with a linear combination of the $\mathrm{Ti}_{2,3}$ edges of $\mathrm{LaTiO}_{3}$ and $\mathrm{SrTiO}_{3}$. The analysis based on these data shown in Fig. 4B of our manuscript indicates that the fractional contribution at the $\mathrm{LaO}$ layer is $24 \% \mathrm{for}^{3 i^{3+}}$ while $76 \%$ for $\mathrm{Ti}^{4+}$. The decay of the $\mathrm{Ti}^{3+}$ and $\mathrm{La}$ signal from the $\mathrm{LaO}$ layer into the $\mathrm{SrTiO}_{3}$ film was fit with the Lorentzian distribution, yielding an $\sim 1 \mathrm{~nm}$ spatial distribution (full width at half maximum) of $\mathrm{Ti}^{3+}$ and $\mathrm{La}$ across the LaO layer (see Fig. S5, S6)

\section{Synchrotron diffraction measurements}

Room temperature synchrotron x-ray scattering measurements of the 10 uc $\mathrm{SrTiO}_{3} / 1 \mathrm{ML}$ $\mathrm{LaO} / \mathrm{SrTiO}_{3}$ and 10 uc $\mathrm{SrTiO}_{3} / 1 \mathrm{ML} \mathrm{SmO} / \mathrm{SrTiO}_{3}$ heterostructures were performed at Sector 33- 
BM of the Advanced Photon Source. In-plane (K) scans along the [010] direction are shown in Fig. S6, where reciprocal lattice units are referenced to the $\mathrm{SrTiO}_{3}$ lattice parameter at room temperature. As seen, both samples exhibit sharp half-order peaks, indicating the presence of a structure with $2 \times 2 \times 2$ unit cell periodicity with respect to the $\mathrm{SrTiO}_{3}$ substrate, as expected for perovskites with tilted oxygen octahedra (S18). While $1 / 2\{$ odd, odd, odd $\}$ peaks were observed for both $\mathrm{H} \neq \mathrm{K}$ and $\mathrm{H}$ or $\mathrm{K} \neq \mathrm{L}$ (such as $1 / 2\{3,1,1\}$ and $1 / 2\{3,3,1\}$, respectively), mixed odd and even integer peaks such as the $1 / 2\{3,0,1\}$ were absent, as were peaks of the form $1 / 2\{H, H, H\}$. Following the structure factor calculations of Ref. S10, this allows us to conclude that the Glazer tilt system is $a^{-} a^{-} c^{-}$, where we have assumed in-plane, biaxial symmetry. This is in good agreement with the results of computational theory. Half-order reflections were not observed from the $\mathrm{SrTiO}_{3}$ substrate, which would produce strong, sharp peaks in positions consistent with bulk $\mathrm{SrTiO}_{3}$.

In-plane rocking curves taken on the $1 / 2\{3,1,1\}$ peaks for the 10 uc $\mathrm{SrTiO}_{3} / 1 \mathrm{ML} \mathrm{LaO} /$ $\mathrm{SrTiO}_{3}$ and $10 \mathrm{uc} \mathrm{SrTiO}_{3} / 1 \mathrm{ML} \mathrm{SmO} / \mathrm{SrTiO}_{3}$ heterostructures are shown in Figure $\mathrm{S} 7$ (a). From the FWHMs, we find the in-plane domain size is $\geq 88 \mathrm{~nm}$ for the LaO sample and $\geq 60 \mathrm{~nm}$ for the SmO sample. Scans along L, the out-of-plane direction, are shown in Figure S7(b). For both samples, the half-order reflections are similarly broad along L, with the FWHM corresponding to a domain width of $\sim 2 \mathrm{~nm}$. 

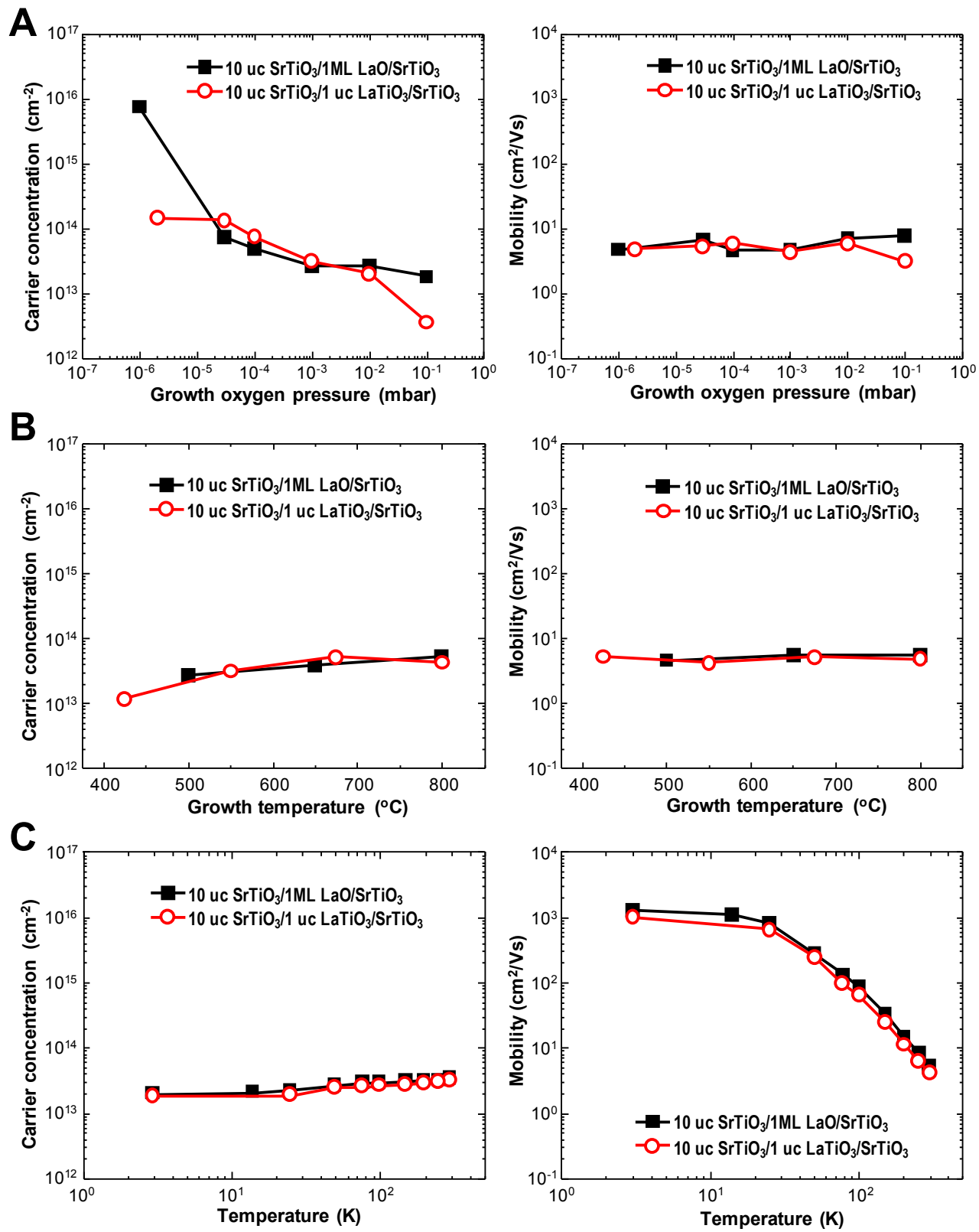

Figure S1. Electrical properties of 10 uc $\mathrm{SrTiO}_{3} / 1 \mathrm{ML} \mathrm{LaO} / \mathrm{SrTiO}_{3}$ and 10 uc $\mathrm{SrTiO}_{3} / 1$ uc $\mathrm{LaTiO}_{3} / \mathrm{SrTiO}_{3}$ heterostructures. (A) Sheet carrier concentration and mobility as a function of growth oxygen partial pressure. The growth temperature was $500{ }^{\circ} \mathrm{C}$. (B) Sheet carrier concentration and mobility as a function of growth temperature. The growth oxygen pressure was $10^{-3}$ mbar. (C) Sheet carrier concentration and mobility as a function of temperature. The growth temperature and oxygen pressure were $500{ }^{\circ} \mathrm{C}$ and $10^{-3} \mathrm{mbar}$, respectively. 

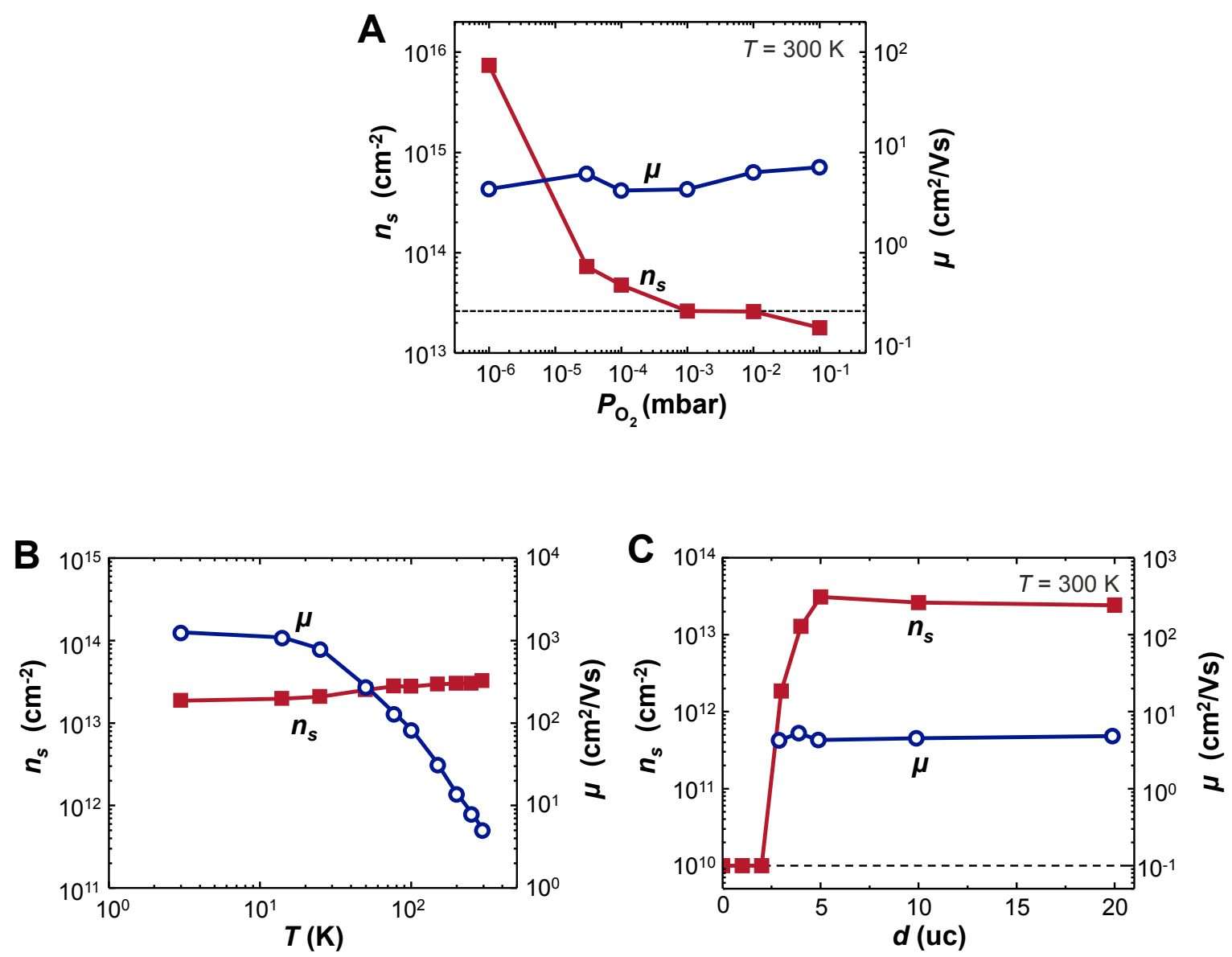

Figure S2. Electrical properties of $\mathrm{SrTiO}_{3} / 1 \mathrm{ML} \mathrm{LaO} / \mathrm{SrTiO}_{3}$ heterostructures. (A) Sheet carrier concentration and mobility as a function of growth oxygen pressure for $10 \mathrm{uc} \mathrm{SrTiO}_{3} / 1 \mathrm{ML}$ $\mathrm{LaO} / \mathrm{SrTiO}_{3}$ heterostructures. The horizontal dotted line indicates a plateau carrier concentration in the intermediate pressure regime, indicating that the carrier concentration does not depend on oxygen pressure in this regime. (B) Sheet carrier concentration and mobility as a function of temperature for a $10 \mathrm{uc} \mathrm{SrTiO}_{3} / 1 \mathrm{ML} \mathrm{LaO} / \mathrm{SrTiO}_{3}$ heterostructure grown at $P_{\mathrm{O}_{2}}=10^{-3}$ mbar. (C) Sheet carrier concentration and mobility as a function of the thickness of $\mathrm{SrTiO}_{3}$ overlayer for $\mathrm{SrTiO}_{3} / 1 \mathrm{ML} \mathrm{LaO} / \mathrm{SrTiO}_{3}$ heterostructures grown at $P_{O_{2}}=10^{-3}$ mbar. The measurement limit in sheet carrier concentration indicated by the horizontal dashed line is $10^{10} \mathrm{~cm}^{-2}$. 
A

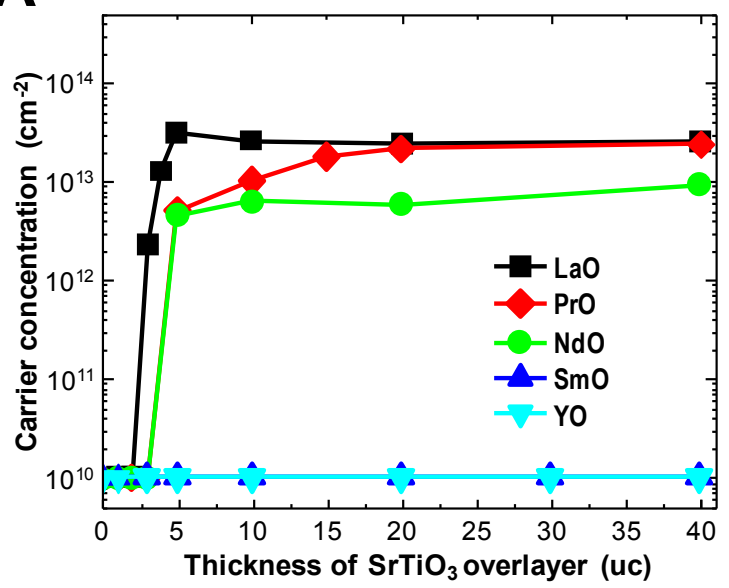

C

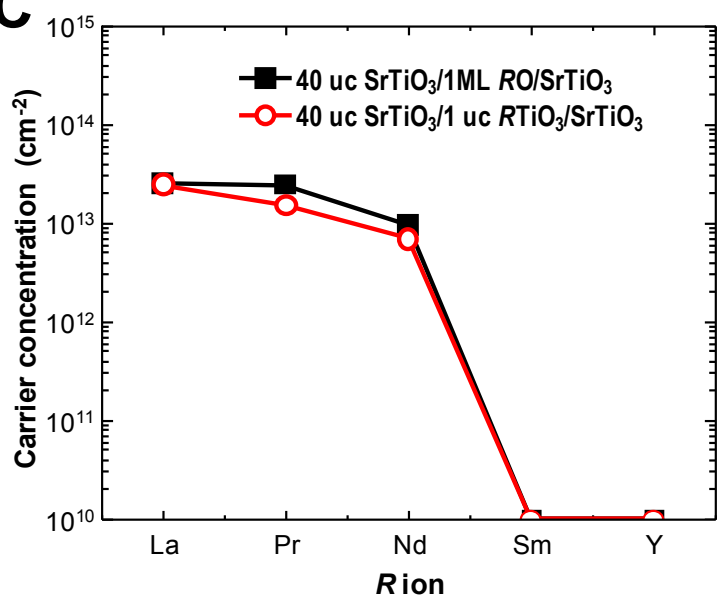

B

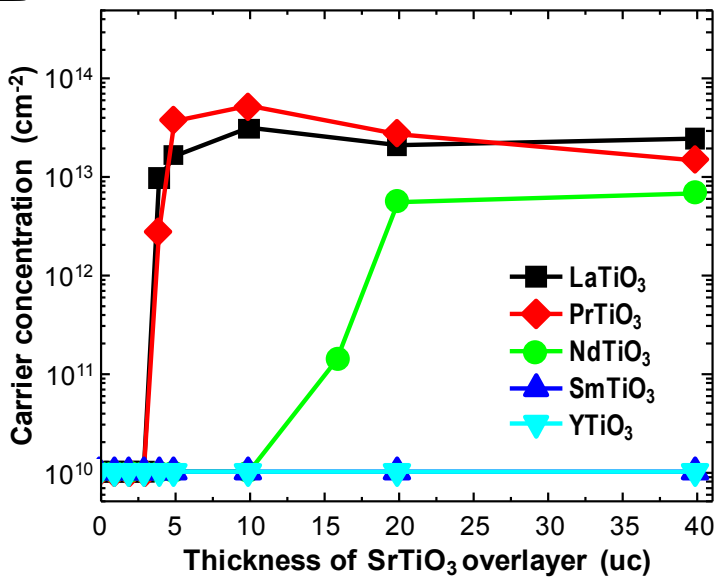

D

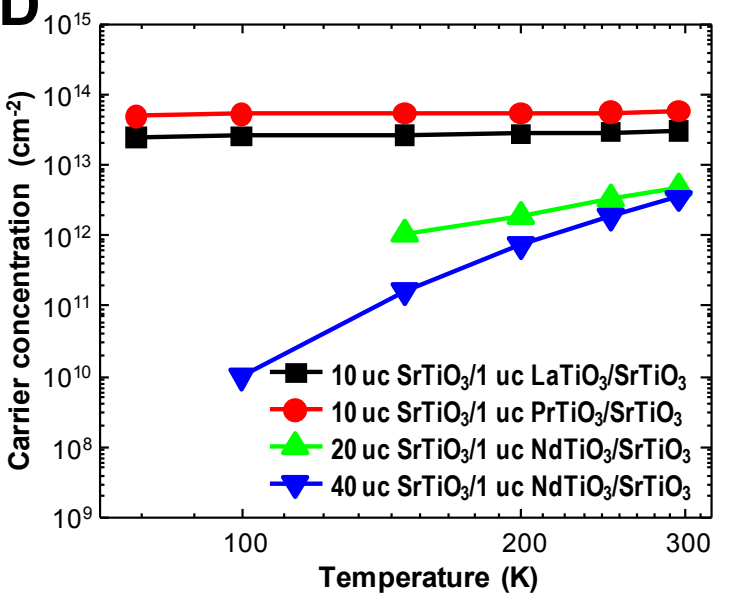

Figure S3. Dependence of sheet carrier concentration on the $R(=\mathrm{La}, \mathrm{Pr}, \mathrm{Nd}, \mathrm{Sm}, \mathrm{Y})$ ion in $\mathrm{SrTiO}_{3} / 1 \mathrm{ML} \quad R \mathrm{O} / \mathrm{SrTiO}_{3}$ and $\mathrm{SrTiO}_{3} / 1$ uc $R \mathrm{TiO}_{3} / \mathrm{SrTiO}_{3}$ heterostructures. (A) Sheet carrier concentration as a function of the thickness of the $\mathrm{SrTiO}_{3}$ overlayer in $\mathrm{SrTiO}_{3} / 1 \mathrm{ML} R \mathrm{O} / \mathrm{SrTiO}_{3}$ heterostructures. (B) Sheet carrier concentration as a function of the thickness of the $\mathrm{SrTiO}_{3}$ overlayer in $\mathrm{SrTiO}_{3} / 1$ uc $R \mathrm{TiO}_{3} / \mathrm{SrTiO}_{3}$ heterostructures. (C) Sheet carrier concentration as a function of $R \mathrm{O}$ or $R \mathrm{TiO}_{3}$ doping layers. (D) Temperature dependence of sheet carrier concentration in conducting $\mathrm{SrTiO}_{3} / 1$ uc $R \mathrm{TiO}_{3} / \mathrm{SrTiO}_{3}$ heterostructures. $\mathrm{NdTiO}_{3}$-based heterostructures show semiconducting behavior, whereas $\mathrm{LaTiO}_{3^{-}}$and $\mathrm{PrTiO}_{3}-\mathrm{based}$ heterostructures show metallic behavior. 

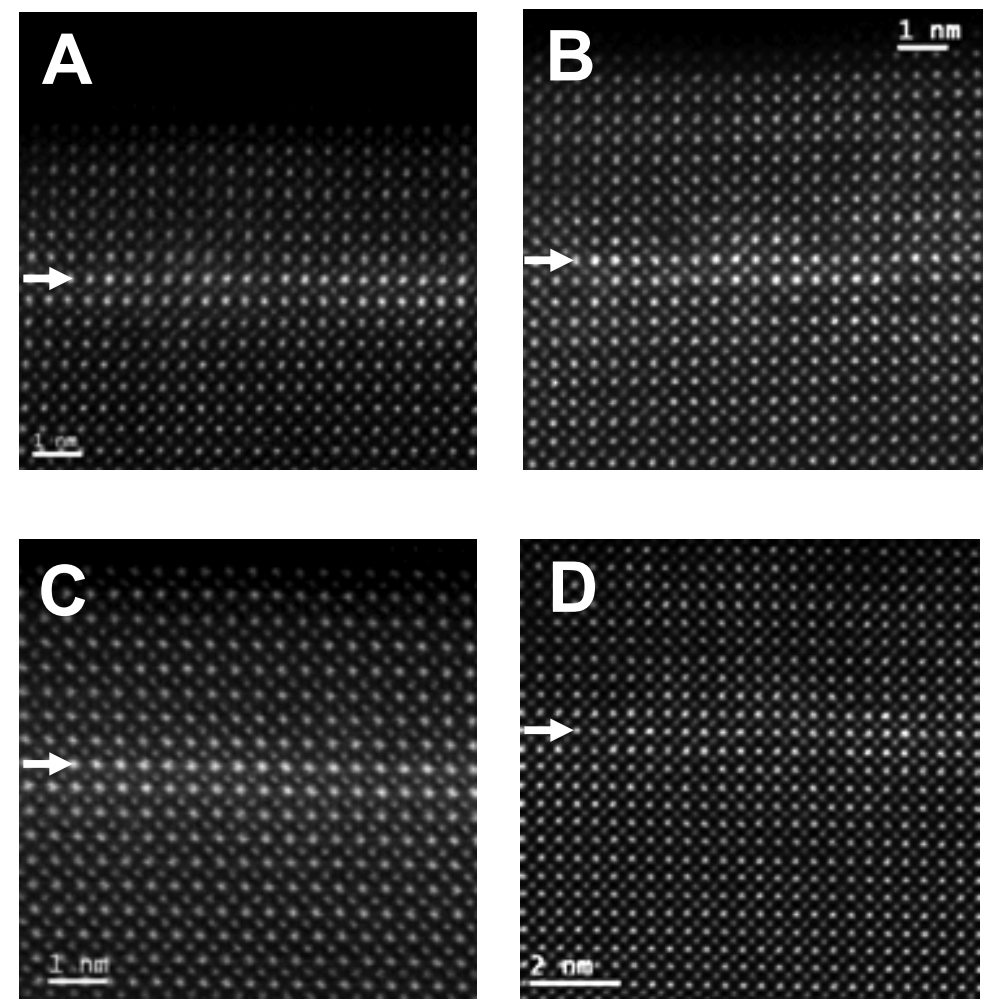

Figure S4. HAADF images of (A) 10 uc $\mathrm{SrTiO}_{3} / 1 \mathrm{ML} \mathrm{LaO}_{/} / \mathrm{SrTiO}_{3}$, (B) 10 uc $\mathrm{SrTiO}_{3} / 1 \mathrm{ML}$ $\mathrm{SmO} / \mathrm{SrTiO}_{3}$, (C) 10 uc $\mathrm{SrTiO}_{3} / 1$ uc $\mathrm{LaTiO}_{3} / \mathrm{SrTiO}_{3}$, and (D) 10 uc $\mathrm{SrTiO}_{3} / 1$ uc $\mathrm{SmTiO}_{3} / \mathrm{SrTiO}_{3}$ heterostructures. The arrows indicate the interfacial $\mathrm{LaO}$ or SmO layers. 

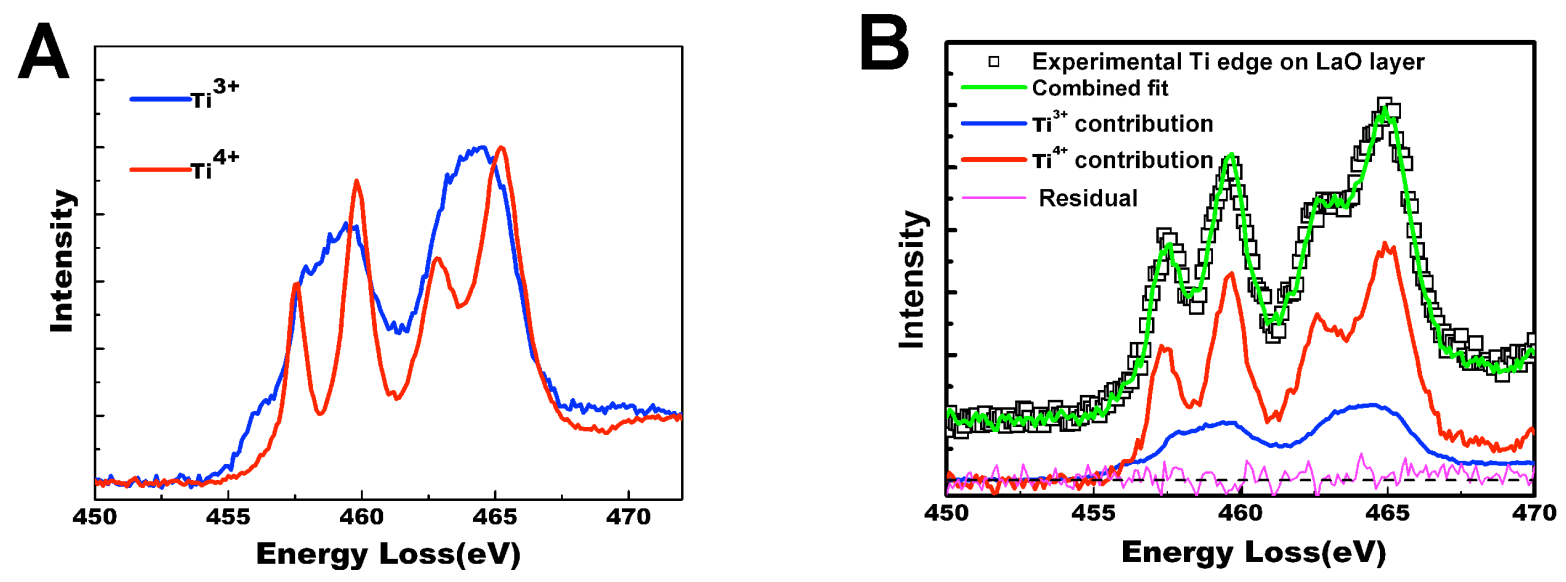

Figure S5 (A.) Ti $\mathrm{L}_{2,3}$ EELS spectrum acquired from the reference sample of $\mathrm{LaTiO}_{3}\left(\mathrm{blue}, \mathrm{Ti}^{3+}\right.$ ) and $\mathrm{SrTiO}_{3}\left(\mathrm{red}, \mathrm{Ti}^{4+}\right)$ with the same sample thickness and same experimental conditions: Convergent angle $=28 \mathrm{mrad}$, collection angle $=20 \mathrm{mrad}$, energy dispersion $=0.1 \mathrm{eV} / \mathrm{ch}$ and probe current $=60 \mathrm{pA}$.

(B) : Quantification of the Ti valence state at the interface ( $\mathrm{LaO}$ layer), as shown in Fig. 4 of the text. The solid blue line is from the reference $\mathrm{Ti}^{3+}\left(\mathrm{LaTiO}_{3}\right)$ and the red line from the reference $\mathrm{Ti}^{4+}\left(\mathrm{SrTiO}_{3}\right)$. The black open-squares are the experimental measurement from the $\mathrm{LaO}$ layer in a $10 \mathrm{uc} \mathrm{SrTiO}_{3} / 1 \mathrm{ML} \mathrm{LaO} / \mathrm{SrTiO}_{3}$ heterostructure and the solid green line is the weighted linear combination of $\mathrm{Ti}^{3+}$ and $\mathrm{Ti}^{4+}$ reference spectra to derive the fractional contribution (24\%) of the $\mathrm{Ti}^{3+}$ state at the interface. The difference spectrum, or the residual to the fit, is also included below (solid pink-line). 


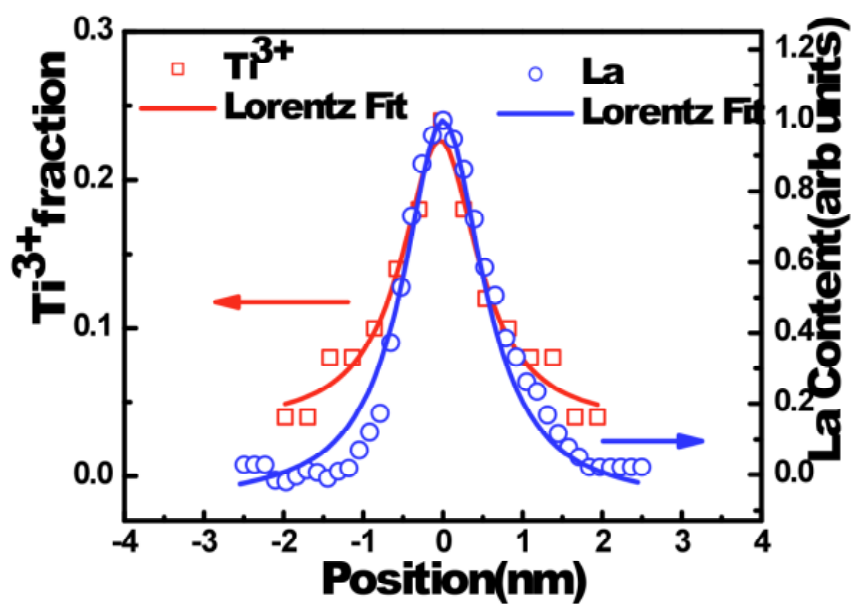

Figure S6: Fractional contribution of $\mathrm{Ti}^{3+}$ from the $\mathrm{LaO}$ layer: open-squares are the measurement and solid-line is the fitting with the Lorentzian distribution, and decay of the $\mathrm{Ti}^{3+}$ and $\mathrm{La}$ signal acquired from the $\mathrm{Ti} \mathrm{L}_{2,3}$ and $\mathrm{La} \mathrm{M}_{4,5}$ edges from the $\mathrm{LaO}$ layer ( $0 \mathrm{~nm}$ position). Both curves were fit with the Lorentizan distribution (solid lines). 


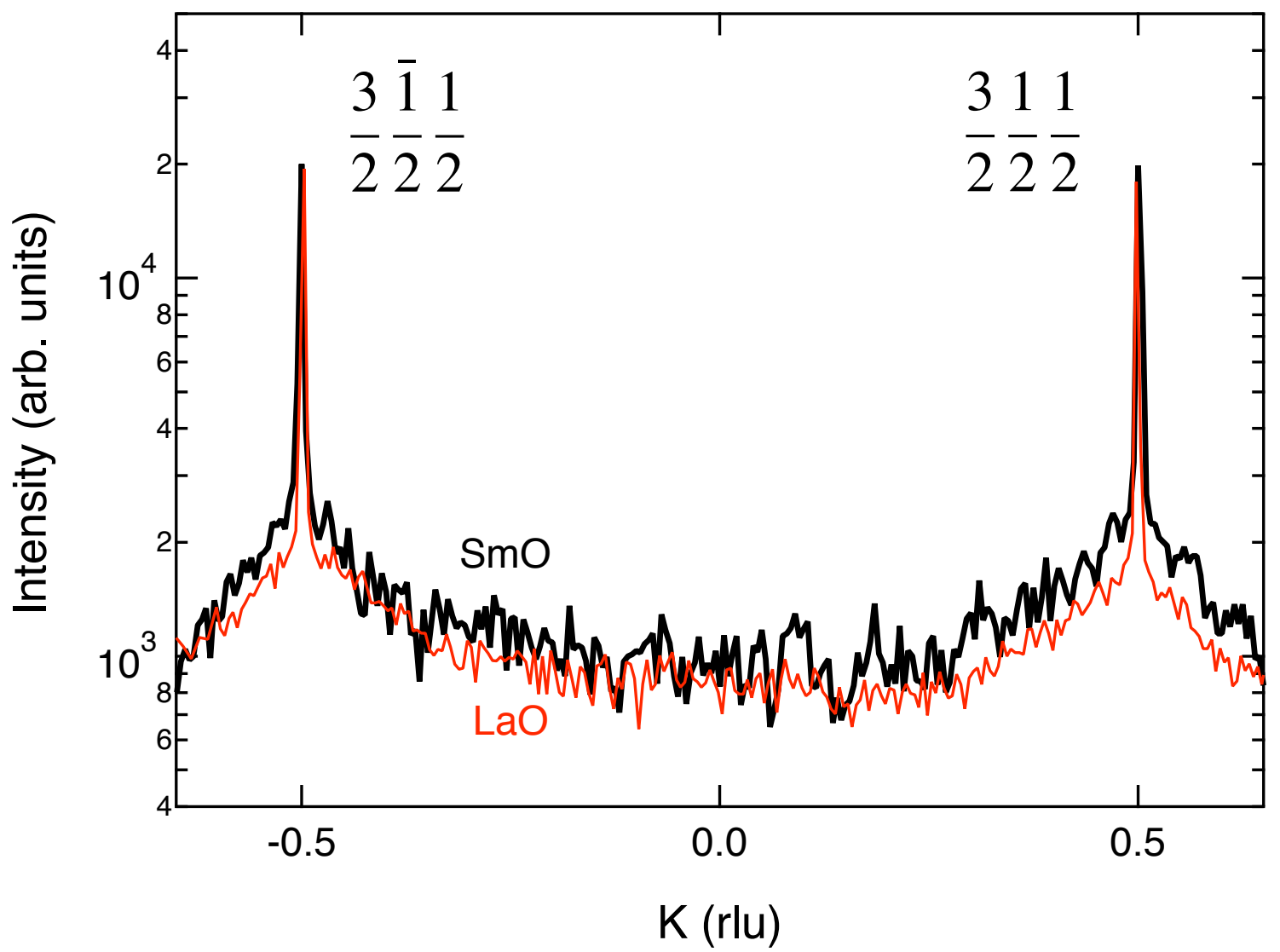

Figure S7. Scattered intensity as function of $\mathrm{K}$, an in-plane direction, with $\mathrm{H}=3 / 2$ and $\mathrm{L}=1 / 2$ for the 10 uc $\mathrm{SrTiO}_{3} / 1 \mathrm{ML} \mathrm{LaO} / \mathrm{SrTiO}_{3}$ and 10 uc $\mathrm{SrTiO}_{3} / 1 \mathrm{ML} \mathrm{SmO} / \mathrm{SrTiO}_{3}$ heterostructures. 

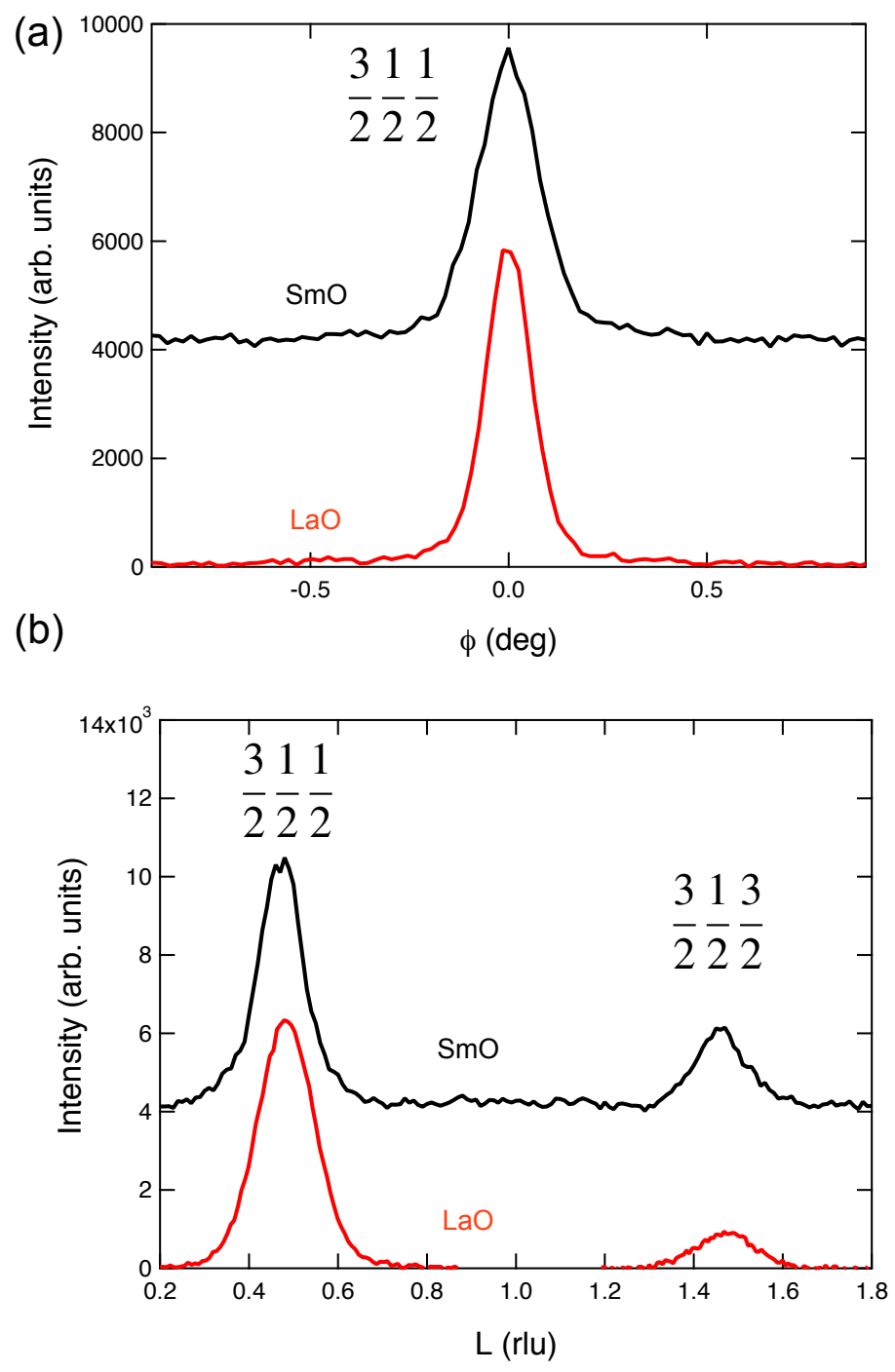

Figure S8. (a) In-plane rocking curves on the $3 / 21 / 21 / 2$ peak for $10 \mathrm{uc} \mathrm{SrTiO}_{3} / 1 \mathrm{ML}$ $\mathrm{LaO} / \mathrm{SrTiO}_{3}$ (red) and $10 \mathrm{uc} \mathrm{SrTiO}_{3} / 1 \mathrm{ML} \mathrm{SmO} / \mathrm{SrTiO}_{3}$ (black) heterostructures. (b) Scattered intensity as function of $\mathrm{L}$, the out-of-plane direction, with $\mathrm{H}=3 / 2$ and $\mathrm{K}=1 / 2$. The intensity of the $\mathrm{SmO}$ heterostructure (black) is offset from that of the $\mathrm{LaO}$ (red). The reciprocal lattice units (rlu) are based on room-temperature cubic $\mathrm{SrTiO}_{3}(a=3.905 \AA)$. 
Table S1. Lattice parameters of $\mathrm{SrTiO}_{3}, \mathrm{LaTiO}_{3}, \mathrm{PrTiO}_{3}, \mathrm{NdTiO}_{3}, \mathrm{SmTiO}_{3}$, and $\mathrm{YTiO}_{3} . a, b, c$ are orthorhombic lattice parameters, and $a_{p c}$ and $b_{p c}$ are pseudocubic. $\mathrm{LaTiO}_{3}, \mathrm{PrTiO}_{3}$, and $\mathrm{NdTiO}_{3}$ on $\mathrm{SrTiO}_{3}$ substrates are biaxially compressive, and $\mathrm{SmTiO}_{3}$ and $\mathrm{YTiO}_{3}$ on $\mathrm{SrTiO}_{3}$ substrates are biaxially tensile.

\begin{tabular}{cccccccc}
\hline \hline Materials & $a(\AA)$ & $b(\AA)$ & $c(\AA)$ & $a_{p c}(\AA)$ & $b_{p c}(\AA)$ & $\begin{array}{c}\text { Averaged } \\
a_{p c}(\AA)\end{array}$ & $\begin{array}{c}\text { Biaxial strain } \\
\text { on } \mathrm{SrTiO}_{3}(\%)\end{array}$ \\
\hline $\mathrm{SrTiO}_{3}$ & & & & 3.905 & 3.905 & 3.905 & 0 \\
$\mathrm{LaTiO}_{3}$ & 5.595 & 5.604 & 7.906 & 3.959 & 3.953 & 3.956 & -1.29 \\
$\mathrm{PrTiO}_{3}$ & 5.555 & 5.615 & 7.830 & 3.949 & 3.915 & 3.932 & -0.69 \\
$\mathrm{NdTiO}_{3}$ & 5.524 & 5.657 & 7.793 & 3.952 & 3.897 & 3.924 & -0.50 \\
$\mathrm{SmTiO}_{3}$ & 5.468 & 5.665 & 7.737 & 3.934 & 3.869 & 3.902 & 0.09 \\
$\mathrm{YTiO}_{3}$ & 5.341 & 5.686 & 7.621 & 3.893 & 3.811 & 3.852 & 1.38 \\
\hline \hline
\end{tabular}




\section{References}

S1. A. Kalabukhov et al., Phys. Rev. B 75, 121404 (2007).

S2. G. Herranz et al., Phys. Rev. Lett. 98, 216803 (2007).

S3. M. Basletic et al., Nat. Mater. 7, 621 (2008).

S4. C. Bell, S. Harashima, Y. Hikita, H. Y. Hwang, Appl. Phys. Lett. 94, 222111 (2009).

S5. W. Siemons et al., Phys. Rev. Lett. 98, 196802 (2007).

S6. A Brinkman et al., Nature Mater. 6, 493 (2007).

S7. S. Thiel, G. Hammerl, A. Schmehl, C. W. Schneider, J. Mannhart, Science 313, 1942 (2006).

S8. Z. S. Popović, S. Satpathy, R. M. Martin, Phys. Rev. Lett. 101, 256801 (2008).

S9 W. Kohn and L. J. Sham, Phys. Rev. 140, A1133 (1965).

S10 P. E. Blöchl, Phys. Rev. B 50, 17953 (1994).

S11 G. Kresse and J. Furtmuller, Phys. Rev. B 54, 11169 (1996).

S12 V. I. Anisimov, F. Aryasetiawan and A. I. Lichtenstein, J. Phys. Condens. Matter 9, 767 (1997).

S13 S. Okatov, A. Poteryaev, and A. Lichtenstein, Europhys. Lett. 70, 499 (2005).

S14 I. V. Solovyev, Phys. Rev. B 74, 054412 (2006).

S15 Z. Zhong and P. J. Kelly, Europhys. Lett., 84, 27001 (2008)

S16 S. Okamoto, A. J. Millis, N. A. Spaldin, Phys. Rev. Lett. 97, 056802 (2006)

S17 R. Pentcheva and W. E. Pickett, Phys. Rev. Lett. 99, 016802 (2007)

S18 A. M. Glazer, Acta Cryst. A31, 756 (1975). 